\title{
Real-time Selective Harmonic Minimization using a Hybrid Analog/Digital Computing Method
}

This paper was downloaded from TechRxiv (https://www.techrxiv.org).

LICENSE

CC BY 4.0

SUBMISSION DATE / POSTED DATE

$13-10-2021 / 26-10-2021$

CITATION

Poon, Jason (2021): Real-time Selective Harmonic Minimization using a Hybrid Analog/Digital Computing Method. TechRxiv. Preprint. https://doi.org/10.36227/techrxiv.16807069.v1

DOI

10.36227/techrxiv.16807069.v1 


\title{
Real-time Selective Harmonic Minimization using a Hybrid Analog/Digital Computing Method
}

\author{
Jason Poon, Member, IEEE, Mohit Sinha, Member, IEEE, \\ Sairaj Dhople, Member, IEEE, and Juan Rivas-Davila, Senior Member, IEEE
}

\begin{abstract}
We present a hybrid analog/digital computing circuit to solve a selective harmonic minimization problem. The approach leverages favorable attributes of digital and analog controllers to yield a fast and scalable optimization solver. A digital microcontroller programs the cost function and other user-defined inputs to the optimization. Voltages in the circuit represent switching angles in the optimization problem. In steady state, the voltages converge to Karush-Kuhn-Tucker (KKT) points of the problem. We present a specific realization of the computing circuit that solves for eight independent switching angles for a quarter-wave symmetric PWM driven two-level single-phase inverter. Seven undesired harmonics are minimized while retaining control over the modulation index. The proposed computing circuit is verified with simulations and a PCB hardware implementation. The experimental results demonstrate that the proposed circuit can converge to the optimal solution in less than $5.0 \mathrm{~ms}$, which is substantially faster than existing methods and facilitates real-time implementation. Moreover, the steady-state power consumption of the PCB implementation is approximately $750 \mathrm{~mW}$, which is also significantly lower than published methods for comparable applications. The computing circuit is utilized to generate the PWM for a $2 \mathrm{~kW}$ single-phase inverter, which validates its feasibility in practical applications.
\end{abstract}

Index Terms-Analog computers, dc-ac power converters, optimization methods, power conversion harmonics, selective harmonic minimization.

\section{INTRODUCTION}

$\mathbf{N}$ ONLINEAR programming (NLP) is an important method used in a variety of power and energy systems to solve optimization problems in which the objective function or constraints are nonlinear. Some examples of NLP-based control problems include model predictive control [2]-[4], optimal power flow [5], [6], state and parameter estimation [7], and motor control [8], [9]. Another widely studied NLP-based control problem is selective harmonic minimization, which refers to a broad class of problems of optimally determining the pulse-width modulation (PWM) of a power convertertypically a two-level or multilevel inverter-to precisely control or minimize the output harmonic content [10]-[15]. The technique generally improves harmonic spectra at reduced switching frequency, thereby yielding lower switching losses

This paper was presented in part at the 2021 IEEE Applied Power Electronics Conference and Exposition (APEC), Phoenix, AZ. June 14, 2021 [1]. (Corresponding author: Jason Poon.)

Jason Poon and Juan Rivas-Davila are with the Electrical Engineering Department, Stanford University, Stanford, CA 94305 USA (email: jason.poon; jmrivas@stanford.edu).

M. Sinha is with Enphase Energy (email: msinha@enphaseenergy.com).

S. V. Dhople is with the Electrical and Computer Engineering Department, University of Minnesota, Minneapolis, MN 55455 USA (email: sdhople@umn.edu). and designs with smaller filter components and heat sinks. Several variations of the selective harmonic minimization problem have been put forth for different inverter topologies and applications. For instance, various works consider nonequal dc link voltages in multilevel inverters [16], the symmetry of the PWM waveform (e.g., quarter-wave, half-wave, nonsymmetrical) [17]-[19], and the consideration of cost functions that incorporate harmonic limits set by grid codes [20], [21].

A separate class of work, not considered in this paper, is the selective harmonic elimination problem, wherein a system of transcendental equations are solved to eliminate specific undesired harmonics [22]-[26]. These techniques utilize analytical solutions, approximations, or interpolations of the transcendental equations. These are typically solved on a digital-based microcontroller or field-programmable gate array (FPGA) to facilitate real-time implementation. While the underlying premise of selective harmonic elimination is similar to that of selective harmonic minimization, it does not permit the specification of an objective-based optimization problem (which provides added design and operational flexibility). This constrains its applicability outside settings where elimination of discrete, pre-specified harmonics is the main goal. Further discussions of the similarities and differences between the selective harmonic minimization and selective harmonic elimination problems are available in [14], [15].

Optimization-based selective harmonic minimization problems are typically implemented with pre-computed solutions stored either as a lookup table (LUT) [16]-[21], [27]-[31] or as an artificial neural network (ANN) that is trained offline [32], [33]. (See Table I for an overview of previous approaches.) Many prior works do not provide data regarding the time required to obtain these pre-computed solutions. Typical solution approaches are not tailored for online implementation, and they involve static lookup tables or pre-trained ANNs, which can also consume significant microcontroller resources and memory. For instance, a technique based on a particle swarm optimization [29] has been reported to require $290 \mathrm{~ms}$ to compute a single optimal solution, while other techniques using a genetic algorithm and bee algorithm have been reported to require $700 \mathrm{~s}$ and $10^{3} \mathrm{~s}$, respectively [27]. The response for the full range of anticipated signals and inputs has to be preprogrammed in LUT and ANN applications. This constrains the applicability of the approach to dynamically respond to conditions that are not pre-programmed.

Focusing on addressing limitations of the state-of-the-art methods surveyed above, we present a hybrid analog/digital circuit that solves the selective harmonic minimization problem 
Table I: Comparison of the proposed approach with existing selective harmonic minimization techniques.

\begin{tabular}{|c|c|c|c|c|c|c|c|c|}
\hline & This Work & {$[27]$} & [16] & {$[28]$} & [29] & {$[30]$} & {$[31]$} & {$[32]$} \\
\hline Number of angles optimized & 8 & 3 & 3 & 15 & 5 & $3,5,7$ & 6,9 & 5 \\
\hline Optimization method & physics-based computing & BA & SA & SA & PSO & GA & hybrid GA & GA \\
\hline Implementation method & analog/digital circuit & LUT & LUT & LUT & LUT & N/A & LUT & ANN \\
\hline Time to optimal convergence & $<5 \mathrm{~ms}$ & $1000 \mathrm{~s}$ & N/A & N/A & $0.29 \mathrm{~s}$ & N/A & N/A & N/A \\
\hline Real-time optimization? & yes & no & no & no & no & no & no & no \\
\hline
\end{tabular}

$\mathrm{GA}=$ genetic algorithm; $\mathrm{BA}=$ bee algorithm; $\mathrm{SA}=$ simulated annealing; $\mathrm{PSO}=$ particle swarm optimization; $\mathrm{ANN}=$ artificial neural network; LUT $=$ lookup table; N/A = not indicated or implemented

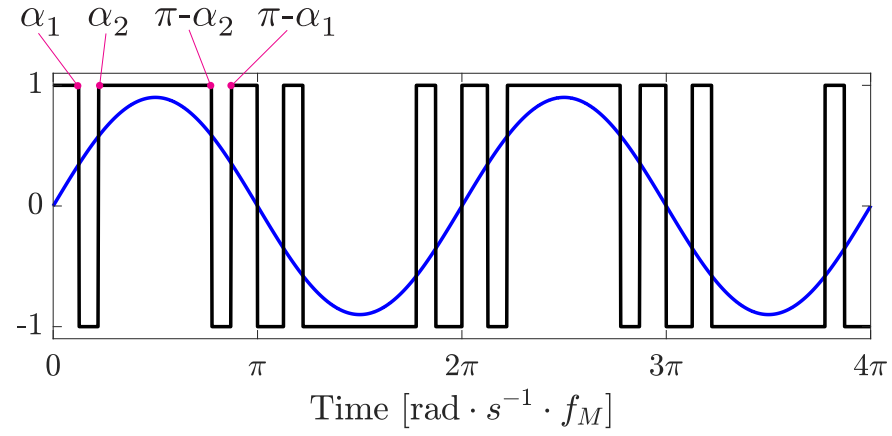

Fig. 1: Illustration of the switching angles $\alpha=\left[\alpha_{1}, \alpha_{2}\right]$ for a quarter-wave symmetric PWM scheme with a fundamental modulation frequency $f_{M}$.

in real-time. To focus ideas, we consider the design and analysis of a computing circuit for a quarter-wave symmetric PWM driven two-level single-phase inverter. Figure 1 illustrates an example PWM waveform with two switching angles. The cost function and desired modulation index is determined by the digital stage of the circuit, and these can be updated in real-time. The voltages in the circuit correspond to the PWM switching angles, and in steady-state, they are feasible solutions to the optimization problem (precisely, they are Karush-Kuhn-Tucker (KKT) points). The proposed computing circuit is verified with a PCB hardware implementation that optimizes eight independent switching angles to eliminate seven undesired harmonics while maintaining control of the modulation index. The hardware implementation demonstrates convergence to the optimal solution in less than $5.0 \mathrm{~ms}$, which is substantially faster than existing methods and facilitates real-time implementation. Moreover, the steady-state power consumption of the PCB implementation is approximately $750 \mathrm{~mW}$, which is also significantly lower than published methods. The computing circuit is utilized to generate PWM signals for a $2 \mathrm{~kW}$ single-phase inverter, which validates its performance and feasibility in practical applications.

The remainder of this paper is organized as follows. Section II presents the analysis and simulation of the hybrid analog/digital computing method for minimizing a single harmonic. Section III presents a generalized realization of the computing circuit for minimizing arbitrary numbers of harmonics while maintaining an arbitrary modulation index. Section IV presents a PCB hardware implementation of the computing circuit that can minimize seven user-selected harmonics in real-time for a two-level single-phase inverter, as well as simulations and experimental measurements that verify the functionality of the hardware implementation. Section V concludes the paper.

\section{A Special Case: Minimizing One Harmonic}

In order to build intuition for the proposed hybrid analog/digital computing method, we consider a special case involving the minimization of a single harmonic for a twolevel inverter subject to a quarter-wave symmetric PWM scheme [30].

\section{A. Problem Formulation}

Consider the PWM switching waveform in Fig. 1 which illustrates switching angles $\alpha=\left[\alpha_{1}, \alpha_{2}\right]$ for a quarter-wave symmetric PWM scheme with a fundamental modulation frequency $f_{M}$. In this example, we will focus on the goal of eliminating the third harmonic. The selective harmonic minimization problem takes the form:

$$
\begin{aligned}
& \min _{\alpha_{1}, \alpha_{2}} \varphi(\alpha)=\left(1+2\left(-\cos \alpha_{1}+\cos \alpha_{2}\right)-m\right)^{2} \\
&+\left(1+2\left(-\cos 3 \alpha_{1}+\cos 3 \alpha_{2}\right)\right)^{2} \\
& \text { s.t. } \quad f_{1}(\alpha)=\alpha_{1} \geq 0,\left(\lambda_{1}\right) \\
& f_{2}(\alpha)=\alpha_{2}-\alpha_{1} \geq 0,\left(\lambda_{2}\right) \\
& f_{3}(\alpha)=-\alpha_{2}+\pi / 2 \geq 0,\left(\lambda_{3}\right) .
\end{aligned}
$$

The constraints $f_{1}(\alpha), f_{2}(\alpha), f_{3}(\alpha)$ ensure the correct ordering and bounds on the switching angles $\left(0 \leq \alpha_{1} \leq \alpha_{2} \leq \frac{\pi}{2}\right)$, and corresponding Lagrange multipliers are denoted by $\lambda_{1}, \lambda_{2}, \lambda_{3}$, respectively. In what follows, we will leverage the notation $f(\alpha)=\left[f_{1}(\alpha), f_{2}(\alpha), f_{3}(\alpha)\right]^{\mathrm{T}}$ and $\lambda=\left[\lambda_{1}, \lambda_{2}, \lambda_{3}\right]^{\mathrm{T}}$.

For a modulation index, $m=0.9$, Fig. 2 depicts the cost function $\varphi(\alpha)$ from (1a) projected on a two-dimensional plane with the constraints in (1b)-(1d) shown as dashed lines. The function is nonconvex, but we can clearly visualize a global minimum. Indeed, obtaining solutions from such visualizations is impossible when scaling the selective harmonic minimization problem to higher dimensions (i.e., when there are more than two switching variables). In general, however, multiple minima may exist, as has been discussed in previous literature (see, e.g., [14], [15]).

The proposed computing circuit physically realizes the Karush-Kuhn-Tucker (KKT) conditions [34]-[36] for the optimization problem in (1). The KKT conditions are first-order necessary conditions to guarantee a local minimum solution; such solutions are called KKT points. We denote these KKT points by $\alpha^{\star}=\left[\alpha_{1}^{\star}, \alpha_{2}^{\star}\right]^{\mathrm{T}}, \lambda^{\star}=\left[\lambda_{1}^{\star}, \lambda_{2}^{\star}, \lambda_{3}^{\star}\right]^{\mathrm{T}}$. The KKT conditions for (1) take the form:

$$
\begin{aligned}
& \frac{\partial \varphi\left(\alpha^{\star}\right)}{\partial \alpha_{\ell}}+\sum_{j} \lambda_{j}^{\star} \frac{\partial f_{j}\left(\alpha^{\star}\right)}{\partial \alpha_{\ell}}=0, \ell=1,2, \\
& f_{j}\left(\alpha^{\star}\right) \geq 0, \lambda_{j}^{\star} \leq 0, \lambda_{j}^{\star} f_{j}\left(\alpha^{\star}\right)=0, j=1,2,3 .
\end{aligned}
$$




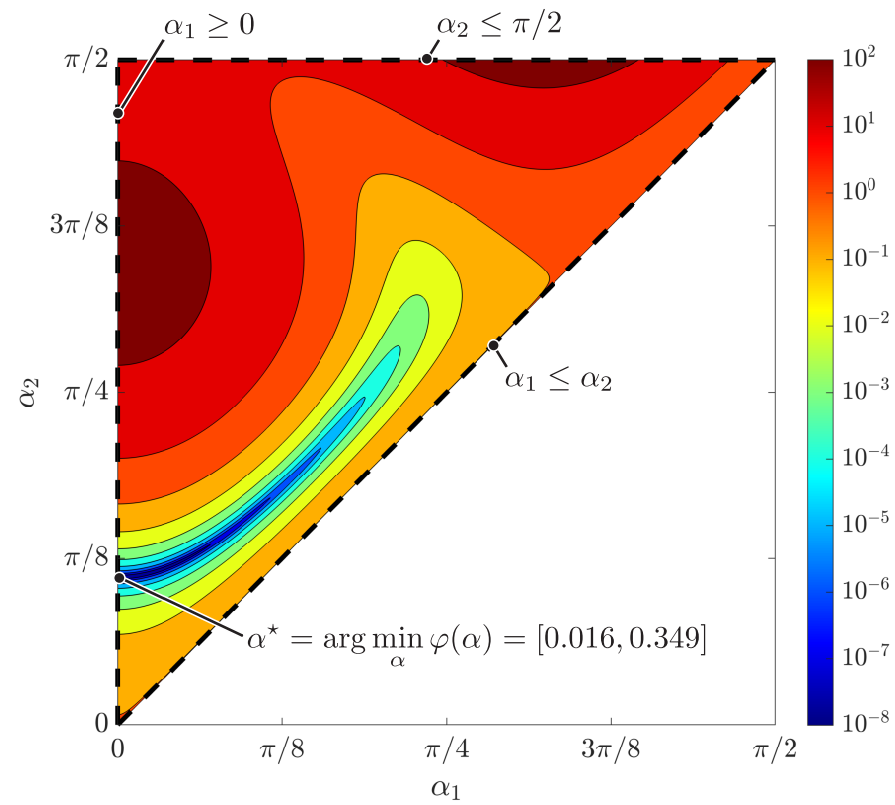

Fig. 2: The cost function $\varphi(\alpha)$ in (1a) plotted as a function of $\alpha_{1}$ and $\alpha_{2}$. The constraints in (1b)-(1d) bound the feasible solution space of $\varphi(\alpha)$. The cost function has a minimum at $\alpha=[0.016,0.349]$.

In particular, (2a) are the so-called stationarity conditions. Furthermore, in (2b), $f_{j}\left(\alpha^{\star}\right) \geq 0$ are primal feasibility conditions, $\lambda_{j}^{\star} \leq 0$ are dual feasibility conditions, and $\lambda_{j}^{\star} f_{j}\left(\alpha^{\star}\right)=0$ are complementary-slackness conditions. Given the constraints in (1b)-(1d), it follows that:

$$
\frac{\partial f_{1}}{\partial \alpha}=[1,0], \frac{\partial f_{2}}{\partial \alpha}=[-1,1], \frac{\partial f_{3}}{\partial \alpha}=[0,-1] .
$$

It is relevant to note that the KKT conditions in (2a) and (2b) have been shown to be continuous or Lipschitz continuous for parametric optimization (the modulation index $m$ being the exogenous parameter in this case) under a set of mild assumptions (see [37]). This is unlike the selective harmonic elimination (SHE) problem, which involves solving harmonic transcendental equations, and have been shown to admit a discontinuous solution space for higher-order multilevel converters (see [38]-[40]).

\section{B. Circuit Realization}

We show next that, with an appropriate mapping and interpretation of the variables $\alpha$ and $\lambda$, the circuit in Fig. 3 yields solutions of the KKT conditions (2a)-(2b). In this circuit, the primal variables, $\alpha_{1}, \alpha_{2}$, and dual variables, $\lambda_{1}, \lambda_{2}, \lambda_{3}$, are voltages at specific nodes; and constraints $f_{1}, f_{2}, f_{3}$ are currents on specific branches. Moreover, note that resistors are labeled with conductances normalized to a $10 \mathrm{k} \Omega$ resistor $\left(R_{\text {nom }}\right)$, and negative resistors are realized by connecting one terminal of the resistor to an inverted output of the equivalent voltage (e.g., the $-\alpha_{\ell}$ terminal in Fig. 4a). The implementation of these inverted voltage rails is straightforward and simply involves an inverting op-amp network.

Notice that the resistive network on the bottom left of the figure encodes the sensitivities of the constraints with respect to the optimization variables (3). Likewise, the resistive network on the top left yields currents that capture the functional form of the constraints in (1b)-(1d). The cost function dynamics of $\varphi(\alpha)$ are introduced to the circuit by timevarying controlled voltage sources with values precisely equal to $\partial \varphi(\alpha) / \partial \alpha_{\ell}$. The implementation of these controlled voltage sources is elaborated on in Section III-B. As we will show through forthcoming developments, the circuit is constructed in a manner such that the steady state values of these voltages and currents precisely satisfy the KKT conditions in (2a) $-(2 b)$. The integrator and nonlinear resistor blocks depicted in Fig. $4 \mathrm{a}$ and Fig. 4b, respectively, are relevant to the operation of the circuit and are explained in detail here.

1) Integrator Block: The integrator block (Fig. 4a) is a transimpedance amplifier with the dynamics of a capacitor, that is, its output is a voltage that is proportional to the integral of the current at its input. The currents flowing into the integrators, $i_{\ell}$, originate from two sources. The first source is the term $\partial \varphi(\alpha) / \partial \alpha_{\ell}$ (from the controlled voltage sources), while the second source is from the sum of terms $\lambda_{j} \partial f_{j}(\alpha) / \partial \alpha_{\ell}$.

2) Nonlinear Resistor Block: The nonlinear resistor (Fig. 4b) is a transimpedance amplifier with a voltage-current relationship shown in Fig. 4 b, specifically:

$$
\lambda=g(\mathcal{I})= \begin{cases}0 & \text { if } \mathcal{I} \leq 0 \\ -\mathcal{I} R & \text { if } \mathcal{I}>0\end{cases}
$$

for some $R$ and where $\mathcal{I}$ is the input current to the nonlinear resistor. A resistor network connects the input of the nonlinear resistors to voltages $\alpha_{1}, \alpha_{2}, \alpha_{3}$ such that the currents flowing into the inputs of the nonlinear resistors are $-f_{1}(\alpha),-f_{2}(\alpha),-f_{3}(\alpha)$.

Having overviewed the subsystems of the circuit realization, we now examine its steady state operation. First, consider that in steady state, the currents $i_{\ell}$ are zero. Given the composition of the currents, we see that this precisely maps to the KKT stationarity condition (2a). Assume that in steady state, $\alpha_{j}$ satisfy the constraints in (1b)-(1d), equivalently, the currents $f_{j}(\alpha)$ satisfy the primal-feasibility constraints in (2b). With negative (or zero) input currents to the nonlinear resistors, the output voltages of the nonlinear resistors are $\lambda_{j}=0$. With this, we see that the voltages $\alpha_{j}, \lambda_{j}$ correspond to the KKT points, $\alpha_{j}^{\star}, \lambda_{j}^{\star}$, i.e., they satisfy all the KKT conditions in (2a), (2b). It is relevant to note that while these KKT points represent local minima of (1), they are not necessarily the global minimum, as finding the global minimum of a nonconvex function such as (1a) is generally an NP-hard problem. Many conventional metaheuristic optimization techniques (e.g., genetic algorithms, particle swarm optimization, among others) introduce noise based on a Gaussian or Boltzmann distribution to the dynamics of the optimization variable to mitigate the likelihood of falling into undesirable local minima (e.g., [41]-[43]). In the simulation and experimental results that follow, we will evaluate the effectiveness of the solution obtained at the KKT points, which will depend on the initial starting condition of the circuit and the presence of noise. We will see that the proposed approach does not fall into undesired local minima and that the targeted harmonics are effectively minimized in the various tested scenarios. We 


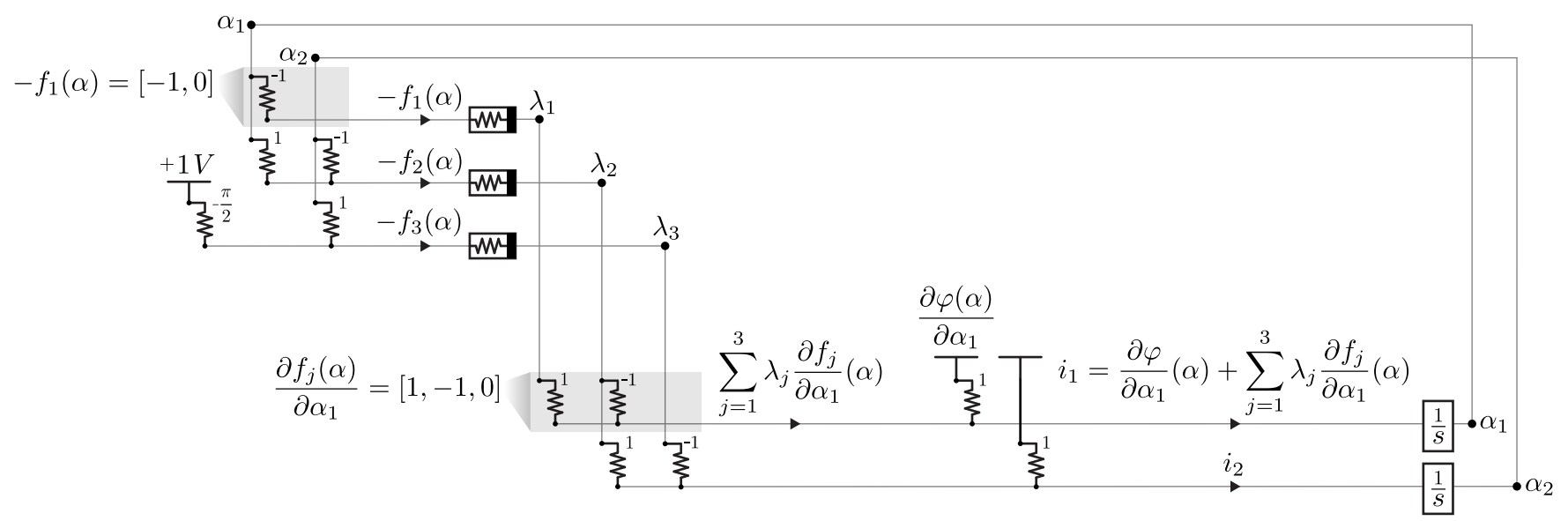

Fig. 3: The circuit implementation of the computing circuit that yields the optimal switching angles $\alpha_{1}^{\star}$ and $\alpha_{2}^{\star}$ that minimize the third harmonic in a two-level inverter. In particular, the circuit minimizes the cost function (1a) subject to constraints (1b)-(1d). The resistors are labeled with conductances normalized to a $10 \mathrm{k} \Omega$ resistor $\left(R_{\mathrm{nom}}\right)$, and negative resistors are realized by connecting one terminal of the resistor to an inverted output of the equivalent voltage (e.g., the $-\alpha_{\ell}$ terminal in Fig. $\left.4 a\right)$.

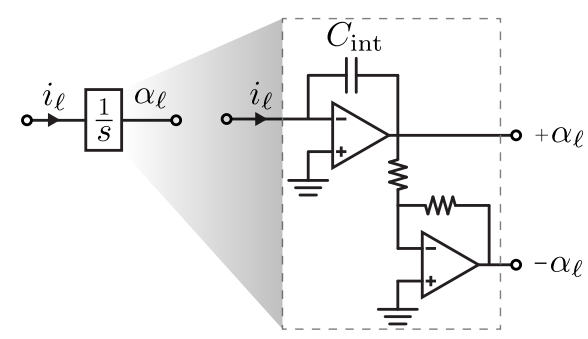

(a) The circuit implementation of the integrator blocks.

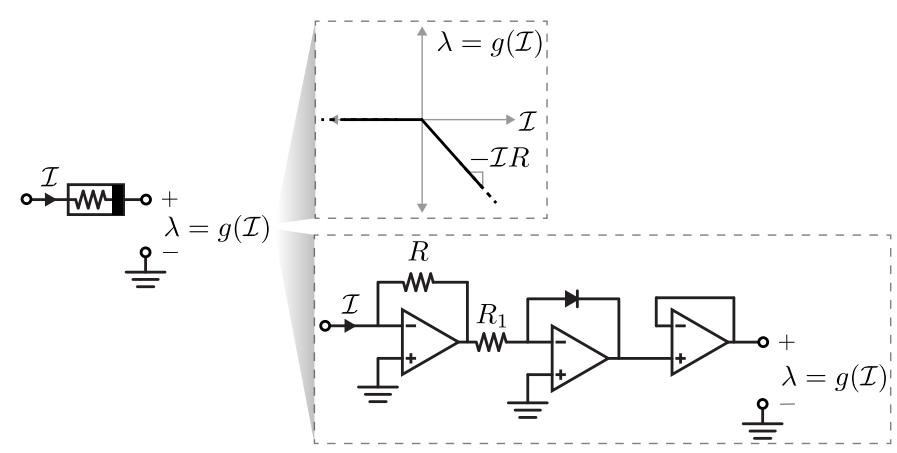

(b) The dynamic behavior and circuit implementation of the nonlinear resistors

Fig. 4: Circuit implementations for elements in the computing circuits in Fig. 3 and Fig. 8.

hypothesize that the analog realization of the proposed circuit may provide a natural noise mechanism, similar to the artificial noise introduced in conventional metaheuristic optimization techniques. Moreover, artificial noise or perturbations could be introduced to dynamics of the optimization variables by the digital microcontroller.

Alternatively, consider the scenario where, e.g., the constraint $f_{1}(\alpha)$ is not satisfied, i.e., the voltage, $\alpha_{1}$ is such that $f_{1}(\alpha)<0$. In this case, the output of the first nonlinear resistor will be a negative voltage $\lambda_{1}=-f_{1}(\alpha) R$. This produces a nonzero current $i_{1}$ at the input of the first integrator, thereby perturbing the $\alpha_{1}$ voltage. In all, the passive nonlinearity in (4) penalizes errors in the constraints $f_{j}(\alpha)$ and perturbs the voltages $\alpha_{j}$ in a direction to satisfy the constraints $f_{j}(\alpha) \geq 0$. The value of the resistance $R$ in the nonlinear resistor block determines the magnitude of the perturbation and, thus, affects the rate of convergence to a steady state solution that satisfies the constraints.

\section{Simulation Results}

The computing circuit depicted in Fig. 3 is simulated using a SPICE-based simulation software. Results are shown in Fig. 5. Figure 5a depicts the evolution of voltages (equivalently, switching angles), $\alpha_{1}, \alpha_{2}$. They approach the steadstate solution $\alpha_{1}^{\star}=0.000099$ and $\alpha_{2}^{\star}=0.349$, in approximately $38 \mu \mathrm{s}$. The simulated $\alpha_{2}^{\star}$ exactly matches the predicted minimum from Fig. 2, while the simulated value of $\alpha_{1}^{\star}$ is approximately the same, albeit with a $1.0 \%$ error within the $[0, \pi / 2]$ variable range. As will be shown, this error imposes no practical consequences on the effectiveness of the solution. The KKT multipliers, $\lambda_{j}$, are plotted in Fig. $5 \mathrm{~b}$. They are driven to zero at around the same instance as the $\alpha_{j}$ convergence. The combination of these results indicates that all KKT conditions in (2a)-(2b) are satisfied in steady state. The resulting PWM time domain waveform is illustrated in Fig. $5 \mathrm{c}$ with the associated switching instances obtained from $\alpha_{1}^{\star}$ and $\alpha_{2}^{\star}$ appropriately labeled. An analysis of this waveform confirms that the modulation index is 0.90 as desired. Note that for this example, there are two switching instances per quarter period, resulting in eight switching instances per period (Fig. 1). However, because the value of $\alpha_{1}^{\star}=0.000099$ is below the minimum on-time of the control logic, two of the switching transitions per period are suppressed. A frequency domain analysis of this waveform is shown in Fig. 5d. The third harmonic is eliminated as desired, while all other odd harmonics remain.

\section{Transient Analysis}

Next, we present a transient analysis of the switching angle and KKT multiplier voltages as a function of the nominal 


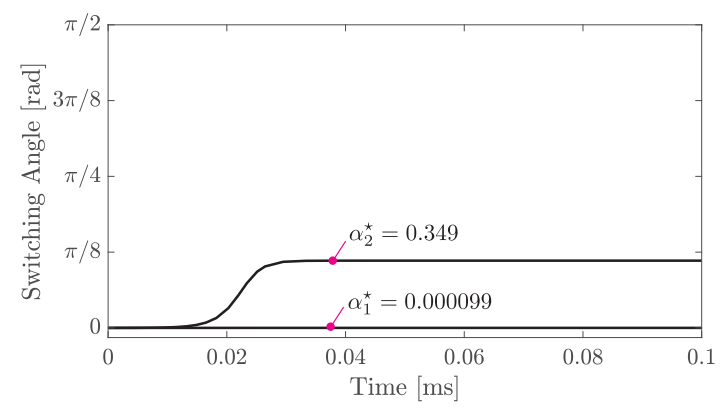

(a) The switching angle voltages $\alpha_{1}$ and $\alpha_{2}$ converge to the optimal value in $38 \mu \mathrm{s}$. These values closely match the expected minimum of the cost function $\varphi(\alpha)$ in Fig. 2

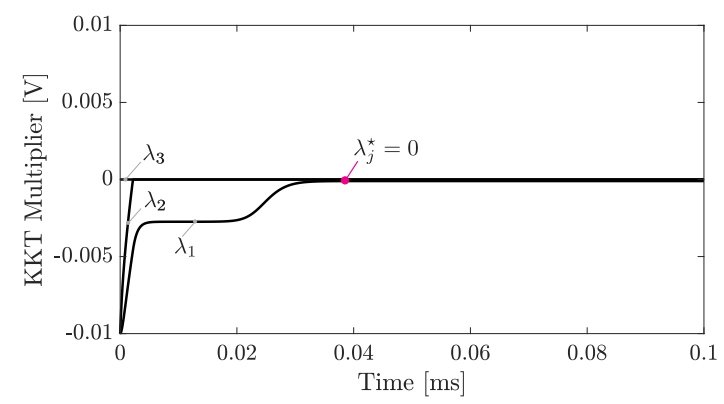

(b) The KKT multiplier voltages become zero in $38 \mu \mathrm{s}$, indicating that the switching angle voltages satisfy the constraints in (1b)-(1d).

$$
\alpha_{1}^{\star} \quad \alpha_{2}^{\star} \pi-\alpha_{2}^{\star} \quad \pi-\alpha_{1}^{\star}
$$

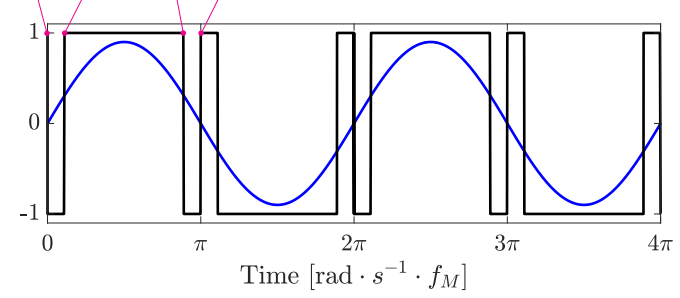

(c) The optimal quarter-wave symmetric two-level waveform obtained by using the computed switching angles $\alpha_{1}^{\star}$ and $\alpha_{2}^{\star}$.

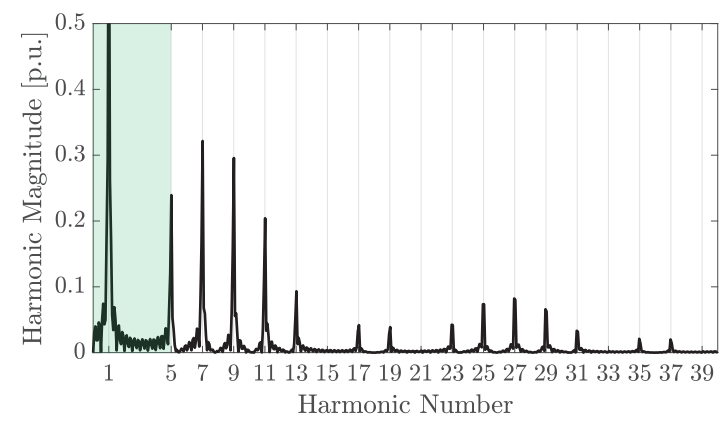

(d) A frequency-domain analysis of the two-level waveform illustrates the minimization of the third harmonic.

Fig. 5: Simulation of the computing circuit minimizing a single odd harmonic.

resistance value $\left(R_{\text {nom }}\right)$ and the integrator capacitance $\left(C_{\text {int }}\right)$. The results of the simulation are illustrated in Figs. 6 and 7. Importantly, as shown, we see that while the values of $R_{\text {nom }}$ and $C_{\mathrm{int}}$ affect the rate of convergence of the switching angles and KKT multiplier voltages in the circuit, they do not affect the final steady state value. Therefore, the steady state operation of the circuit will remain exactly the same. The simulation results illustrate that a larger $R_{\text {nom }}$ and a larger $C_{\text {int }}$ slow the convergence rate of the circuit, while the

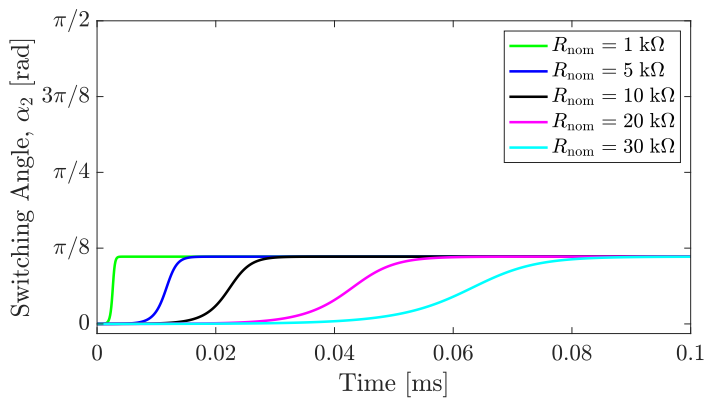

(a) Variations in the convergence of the switching angle voltage $\alpha_{2}$ due to varying $R_{\text {nom }}$.

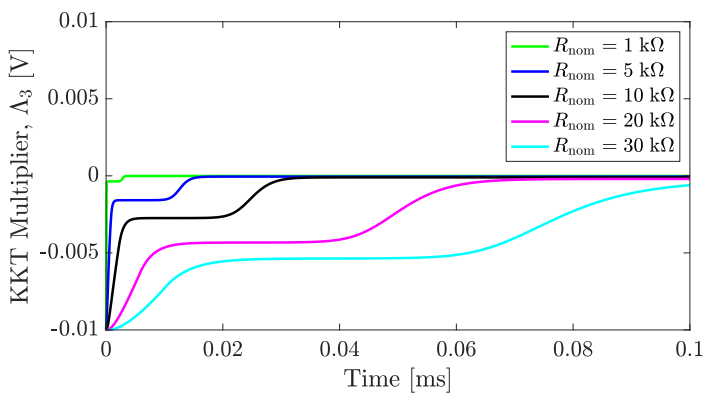

(b) Variations in the convergence of the KKT multiplier voltage $\Lambda_{3}$ due to varying $R_{\text {nom }}$.

Fig. 6: Transient simulation of the proposed computing circuit with variations in the nominal resistance value $R_{\text {nom }}$.

converse has the opposite affect. Thus, the desired rate of convergence can be tuned by selecting $R_{\text {nom }}$ and $C_{\text {int }}$. For example, a larger realization of the circuit may have more parasitics, and will require a slower transient in order to minimize ringing and to maintain the accuracy and stability. Conversely, if a design requires fast convergence, one would attempt to select the smallest possible $R_{\text {nom }}$ and $C_{\text {int }}$ while ensuring that layout parasitics are minimized as well. Such design considerations can be simulated a priori if the parasitics are modeled appropriately in the simulation model.

\section{Generalized Hybrid Analog/Digital COMPUting Circuit for Selective HaRmonic MINIMIZATION}

In this section, we consider the generalized version of the selective harmonic minimization problem leveraging intuition gleaned from the special case of eliminating one harmonic considered in Section II.

\section{A. Problem Formulation}

The generalized version of the problem involves solving for the optimal values of $N$ switching angles to minimize $N-1$ arbitrary harmonics while maintaining an arbitrary modulation index $m$. As before, we consider a quarter-wave symmetric PWM scheme for a two-level inverter. The precise formulation is given by:

$$
\begin{aligned}
\min _{\alpha=\left[\alpha_{1}, \ldots, \alpha_{N}\right]^{\mathrm{T}}} & \varphi(\alpha)=\left(1+2 \sum_{\ell=1}^{N}(-1)^{\ell} \cos \left(\alpha_{\ell}\right)-m\right)^{2} \\
& +\sum_{k=1}^{N-1}\left(1+2 \sum_{\ell=1}^{N}(-1)^{\ell} \cos \left(h_{k} \alpha_{\ell}\right)\right)^{2}
\end{aligned}
$$




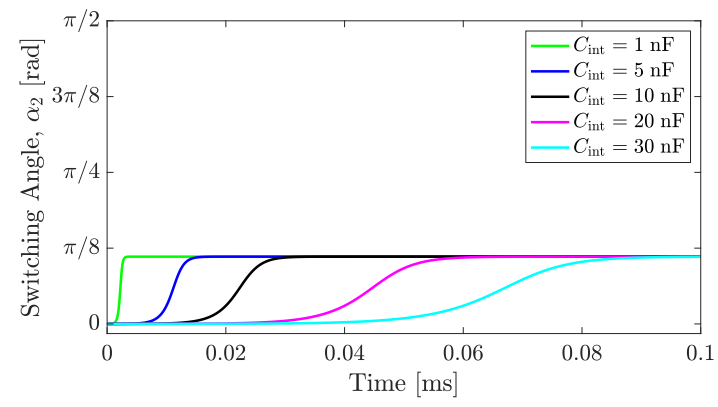

(a) Variations in the convergence of the switching angle voltage $\alpha_{2}$ due to varying $C_{\text {int }}$.

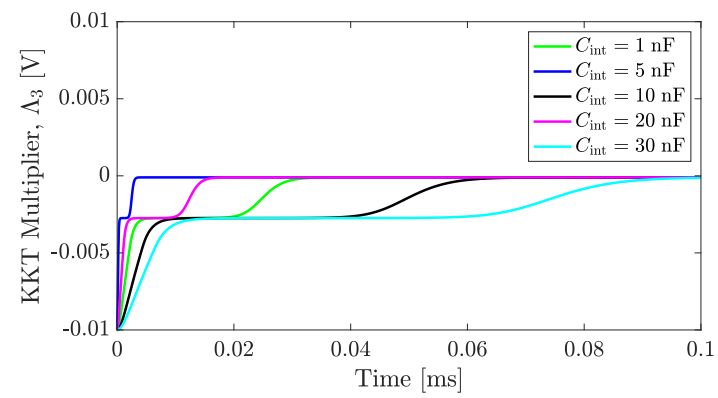

(b) Variations in the convergence of the KKT multiplier voltage $\Lambda_{3}$ due to varying $C_{\text {int }}$.

Fig. 7: Transient simulation of the proposed computing circuit with variations in the integrator capacitance $C_{\text {int }}$.

$$
\begin{aligned}
\text { s.t. } \quad f_{1}(\alpha) & =\alpha_{1} \geq 0,\left(\lambda_{1}\right), \\
f_{2}(\alpha) & =\alpha_{2}-\alpha_{1} \geq 0,\left(\lambda_{2}\right), \\
\vdots & \\
f_{N}(\alpha) & =\alpha_{N}-\alpha_{N-1} \geq 0,\left(\lambda_{N}\right), \\
f_{N+1}(\alpha) & =-\alpha_{N}+\pi / 2 \geq 0,\left(\lambda_{N+1}\right),
\end{aligned}
$$

where $\alpha=\left[\alpha_{1}, \ldots, \alpha_{N}\right]^{\mathrm{T}}$ are the $N$ switching angles to be determined, $\lambda=\left[\lambda_{1}, \ldots, \lambda_{N+1}\right]^{\mathrm{T}}$ are the Lagrange multipliers corresponding to the constraints $f(\alpha)=$ $\left[f_{1}(\alpha), \ldots, f_{N+1}(\alpha)\right]^{\mathrm{T}}$, which enforce $0 \leq \alpha_{1} \leq \cdots \leq$ $\alpha_{N} \leq \frac{\pi}{2}, m$ is the modulation index, and $h_{1}, \ldots, h_{N-1}$ are the orders of the undesired harmonics to be minimized. Depending on the application, $h_{1}, \ldots, h_{N-1}$ are typically chosen to be the lowest odd harmonics (i.e., $3,5,7, \ldots$ ) or, for three-phase applications, the lowest odd nontriplen harmonics (i.e., $5,7,13, \ldots$ ). The KKT conditions for problem (1) take the form:

$$
\begin{aligned}
& \nabla_{\alpha} \varphi\left(\alpha^{\star}\right)+\left(\lambda^{\star}\right)^{\mathrm{T}} \nabla_{\alpha} f\left(\alpha^{\star}\right)=0, \\
& f_{j}\left(\alpha^{\star}\right) \geq 0, \quad \lambda_{j}^{\star} \leq 0, \quad \lambda_{j}^{\star} f_{j}\left(\alpha^{\star}\right)=0, j=1, . ., N+1,
\end{aligned}
$$

where $\nabla_{\alpha} \varphi(\cdot)$ captures sensitivities of the cost function with respect to the optimization variables:

$$
\nabla_{\alpha} \varphi(\alpha)=\left[\begin{array}{lll}
\frac{\partial \varphi(\alpha)}{\partial \alpha_{1}} & \ldots & \frac{\partial \varphi(\alpha)}{\partial \alpha_{N}}
\end{array}\right]
$$

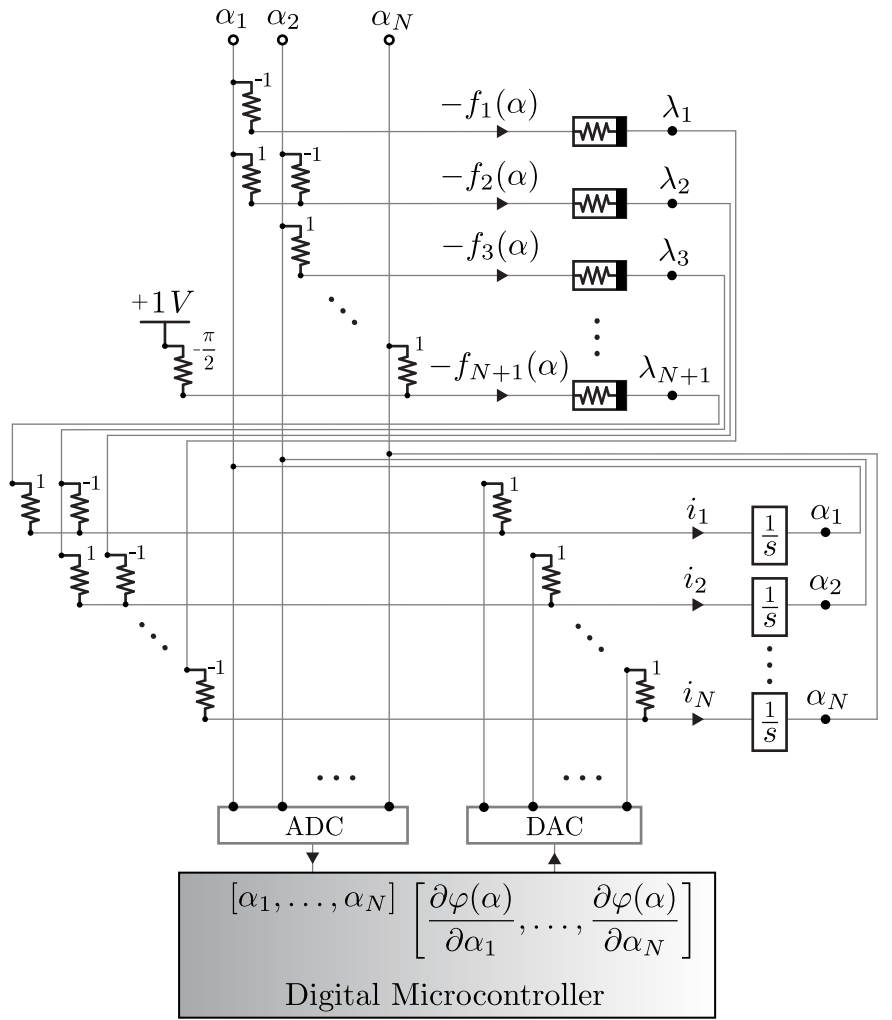

Fig. 8: The circuit implementation of the generalized computing circuit that yields $N$ optimal switching angles $\alpha_{\ell}^{\star}$ that minimize $N-1$ arbitrary harmonics for a two-level inverter. The circuit minimizes the cost function of (5a) with the constraints of (5b). The resistors are labeled with conductances normalized to a $10 \mathrm{k} \Omega$ resistor, and negative resistors are realized by connecting one terminal of the resistor to an inverted output of the equivalent voltage.

and $\nabla_{\alpha} f(\cdot)$ is the Jacobian matrix corresponding to the constraints, given by:

$$
\begin{aligned}
\nabla_{\alpha} f(\alpha) & =\left[\begin{array}{ccccc}
\frac{\partial f_{1}}{\partial \alpha_{1}} & \frac{\partial f_{1}}{\partial \alpha_{2}} & \cdots & \frac{\partial f_{1}}{\partial \alpha_{N}-1} & \frac{\partial f_{1}}{\partial \alpha_{N}} \\
\frac{\partial f_{2}}{\partial \alpha_{1}} & \frac{\partial f_{2}}{\partial \alpha_{2}} & \cdots & \frac{\partial f_{2}}{\partial \alpha_{N-1}} & \frac{\partial f_{2}}{\partial \alpha_{N}} \\
\vdots & \vdots & \ddots & \vdots & \vdots \\
\frac{\partial f_{N}}{\partial \alpha_{1}} & \frac{\partial f_{N}}{\partial \alpha_{2}} & \cdots & \frac{\partial f_{N}}{\partial \alpha_{N-1}} & \frac{\partial f_{N}}{\partial \alpha_{N}} \\
\frac{\partial f_{N+1}}{\partial \alpha_{1}} & \frac{\partial f_{N+1}}{\partial \alpha_{2}} & \cdots & \frac{\partial f_{N+1}}{\partial \alpha_{N-1}} & \frac{\partial f_{N+1}}{\partial \alpha_{N}}
\end{array}\right] \\
= & {\left[\begin{array}{ccccc}
1 & 0 & \cdots & 0 & 0 \\
-1 & 1 & \cdots & 0 & 0 \\
\vdots & \vdots & \ddots & \vdots & \vdots \\
0 & 0 & \cdots & 1 & 0 \\
0 & 0 & \cdots & -1 & 1
\end{array}\right] . }
\end{aligned}
$$

\section{B. Circuit Realization}

Figure 8 illustrates the implementation of the generalized computing circuit to solve the optimization problem (5). In particular, the steady-state values of voltages $\alpha_{1}, \ldots, \alpha_{N}$ and $\lambda_{1}, \ldots, \lambda_{N+1}$ converge to $\alpha_{1}^{\star}, \ldots, \alpha_{N}^{\star}$ and $\lambda_{1}^{\star}, \ldots, \lambda_{N+1}^{\star}$, respectively, which satisfy the KKT conditions $(6 a)-(6 b)$.

The computing circuit merges an analog system composed of a resistor network, integrators (Fig. 4a), and nonlinear resistors (Fig. 4b), with a digital system composed of a small microcontroller and associated analog-to-digital (ADC) and 


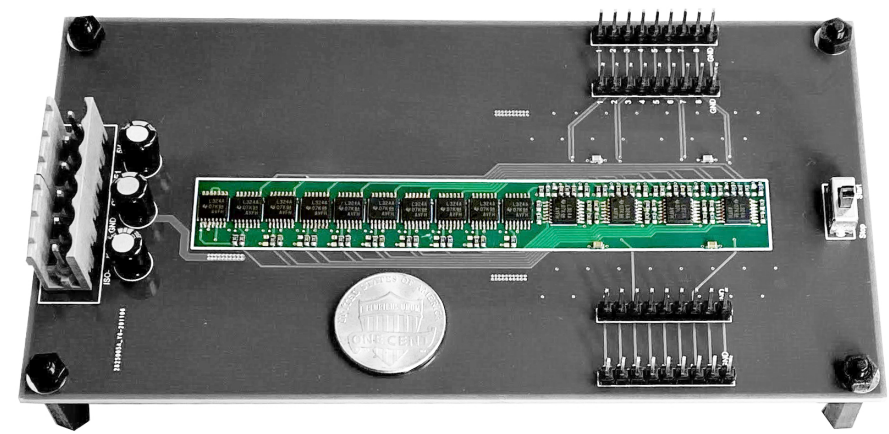

Fig. 9: A photo of the hardware implementation of the proposed computing circuit with a US penny for scale. The active area of the circuit is shown in color and measures $3.925 \times 0.535$ inches.

digital-to-analog converters (DACs). The resistive network on the bottom left of the figure encodes the sensitivities of the constraints with respect to the optimization variables as described by the Jacobian in (8). The resistive network on the top left yields currents that capture the functional form of the constraints in (5b). The microcontroller interfaces with particular nodes in the resistive network through the ADCs and DACs. The microcontroller computes $\partial \varphi / \partial \alpha_{\ell}$ based on measurements of $\alpha_{\ell}$. The programmed cost function includes the harmonics $h_{1}, \ldots, h_{N-1}$ that are to be minimized, along with the modulation index $m$ of the two-level waveform. Notably, these parameters can be changed in real-time and the circuit will dynamically converge to a new solution.

The operation of this circuit is largely similar to that of the realization presented in Section II. In particular, steadystate values of the currents, $i_{\ell}, \ell=1, \ldots, N$ are all zero, and the constitution of these currents implies that this steady-state operating condition satisfies the stationarity condition (6a). Furthermore, the nonlinear resistors operate in concert with the integrators to ensure that voltages, $\lambda_{1}^{\star} \leq 0, \ldots, \lambda_{N+1}^{\star} \leq 0$ and currents $f_{1}\left(\alpha^{\star}\right) \geq 0, \ldots, f_{N+1}\left(\alpha^{\star}\right) \geq 0$.

\section{EXPERIMENTAL VALIDATION}

In this section, we present a PCB implementation of the proposed computing circuit that solves the selective harmonic minimization problem with eight independent switching angles, that is, $N=8$, for an effective switching frequency of $8 \times 4 \times 60 \mathrm{~Hz}=1920 \mathrm{~Hz}$. The PCB implementation, moreover, generates the associated PWM signals to control a $2 \mathrm{~kW}$ single-phase inverter, from which we measure the output voltage to verify the minimization of the seven $(N-1)$ desired harmonics.

\section{A. Hardware Details}

The PCB implementation of the computing circuit is depicted in Fig. 9 with a US penny for scale. The active area of the analog and digital systems of the circuit is shown in color and measures 3.925 by 0.535 inches. The remaining area of the PCB (shown in grayscale) is used for power connectors, voltage measurement test points, signal connectors to the single-phase inverter, and a physical switch for starting the computation or resetting the voltages $\alpha_{\ell}$ to a predefined initial condition.

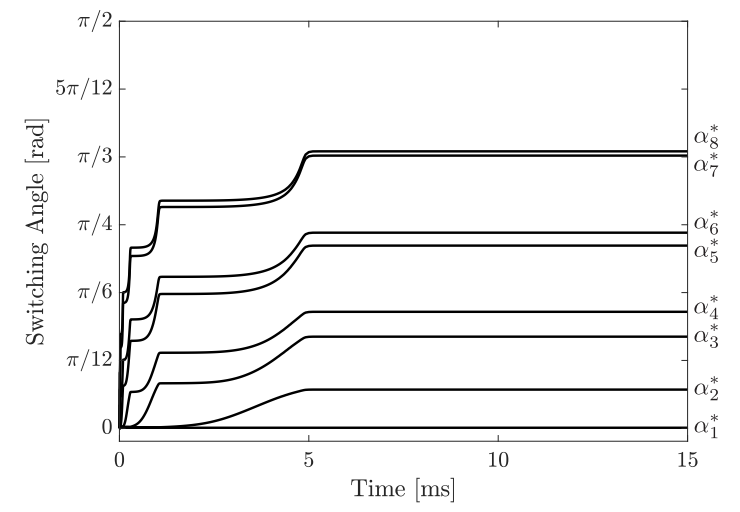

(a) The switching angle voltage variables $\alpha_{\ell}$ converge to the optimal value $\alpha_{\ell}^{\star}$ in approximately $5 \mathrm{~ms}$.

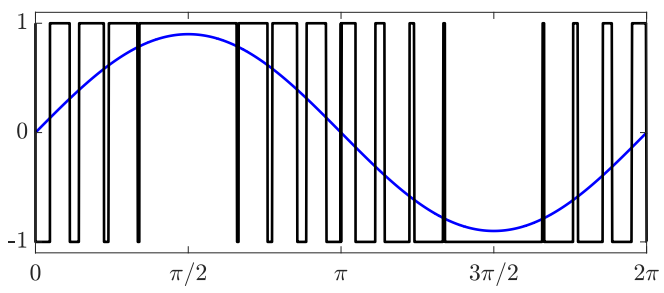

(b) The optimal quarter-wave symmetric two-level waveform obtained by using the computed switching angles $\alpha_{\ell}^{\star}$.

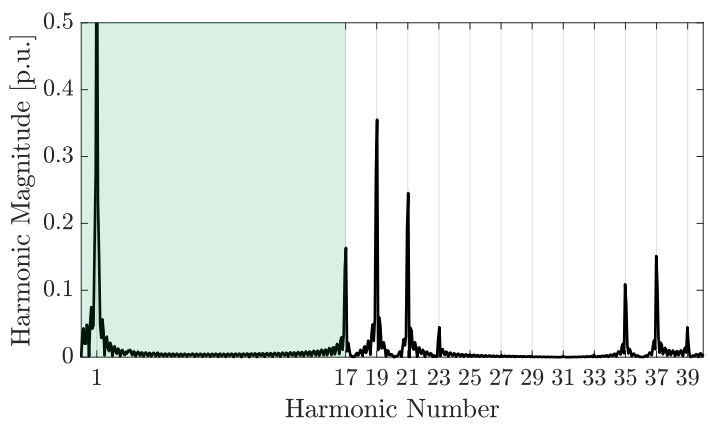

(c) A frequency-domain analysis of the two-level waveform confirms the minimization of the first seven odd harmonics.

Fig. 10: Simulation of the computing circuit minimizing the first seven odd harmonics $(h=3,5,7,9,11,13,15)$.

The analog system is implemented with 14 LM324 general purpose quad op amps, 111 SMD 0603 resistors, 26 SMD 0603 capacitors, and 9 SOT-23 diodes connected as to realize the circuit diagram in Fig. 8. The analog components used in the circuit are generally robust to temperature variations up to $125^{\circ} \mathrm{C}$ and other operating variations, and, moreover, can be implemented using robust automotive grade components if high precision or operation in harsh conditions are required. The analog system of the circuit is 'fixed' (i.e., unchanged between different experiments) for $N=8$ and for the constraints $f_{j} \geq 0$ from (5b). The digital system is implemented with a TI Delphino F28379D microcontroller, its internal ADCs, and an LTC2666 8-channel 16-bit DAC. The microcontroller samples $\alpha_{1}, \ldots, \alpha_{8}$ through the ADC, computes $\partial \varphi / \partial \alpha_{1}, \ldots, \partial \varphi / \partial \alpha_{8}$, and feeds the result back to the analog system through the DAC. The function $\varphi(\alpha)$ is programmed according to the harmonics to be minimized and the desired modulation index, and can be updated in real-time. The microcontroller also contains logic to generate the quarter-wave 
symmetric PWM based on its measurements of $\alpha_{1}, \ldots, \alpha_{8}$. The PWM output is connected to a custom-designed singlephase inverter with a dc link voltage of $400 \mathrm{~V}$ whose output is connected to a $100 \Omega$ resistive load. In steady state, the overall power consumption of the computing circuit, including the analog and digital systems, is approximately $750 \mathrm{~mW}$. While many previous works on selective harmonic minimization do not provide data with respect to power consumption, most metaheuristic optimizers (which many prior techniques utilize) are typically solved on a desktop computer using MATLAB or a similar software, which would consume on the order of 10 's or 100's watts. Therefore, the power consumption of the proposed computing circuit is substantially lower than such techniques. Further study is required to precisely benchmark the power consumption of existing methods used for selective harmonic minimization with the proposed circuit.

\section{B. Verification of Hardware Implementation}

We now present simulation and experimental verification of the hardware implementation discussed above. We conduct three verification tests that involve the minimization of

1) the first seven odd harmonics,

2) the first seven odd nontriplen harmonics, and

3) the first seven odd nontriplen harmonics while the modulation index command, $m$, is time varying.

These verification tests demonstrate the speed of the proposed computing circuit in obtaining the optimal solution, as well as the programmability and generalizability of the implementation to minimize any set of harmonics that are specified by the user. Moreover, the test in which the modulation command is varied intentionally with time demonstrates the ability of the proposed circuit to dynamically respond to inputs and operating conditions that change the optimal solution. This is unique in the literature to the best of the authors' knowledge.

1) Minimization of first seven odd harmonics: In the first verification test, the digital system is programmed to minimize the first seven odd harmonics ( $h=3,5,7,9,11,13,15)$ while maintaining a constant modulation index $m=0.9$. Figure 10 presents results from a SPICE-based simulation of this scenario. As shown in Fig. 10a, the eight switching angle voltage variables $\alpha_{\ell}, \ell=1, \ldots, 8$ from the computing circuit converge in steady state to KKT points, $\alpha_{\ell}^{\star}, \ell=1, \ldots, 8$. Figure $10 \mathrm{~b}$ illustrates one period of the inverter waveform obtained by using these computed switching angles. A frequency domain analysis of this waveform (Fig. 10c) confirms that the first seven odd harmonics are minimized via these KKT points obtained in steady state, $\alpha_{\ell}^{\star}, \ell=1, \ldots, 8$. Figure 11 illustrates measurements from the hardware implementation of this scenario. As shown in Fig. 11a, voltage measurements of $\alpha_{\ell}, \ell=1, \ldots, 8$ from the analog system illustrate that the circuit converges to a steady state value in $5.0 \mathrm{~ms}$. The transient dynamics and steady-state values of $\alpha_{\ell}, \ell=1, \ldots, 8$ closely match the simulation seen in Fig. 10a. The resulting single-phase inverter waveform is shown in Fig. 11b in the time and frequency domains. As shown, the harmonics below $h=17$ are indeed minimized, while odd harmonics remain in the region $h>17$.

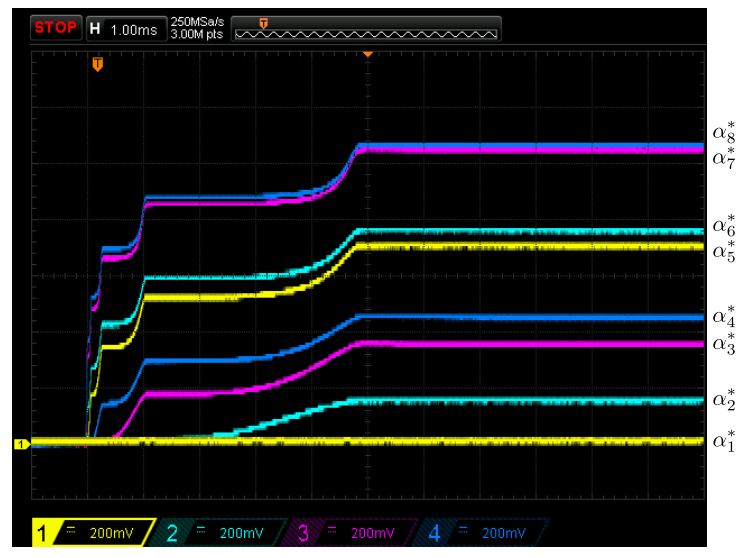

(a) Measurements of the switching angle voltage variables $\alpha_{\ell}$ converge to the optimal value $\alpha_{\ell}^{\star}$ in $5.0 \mathrm{~ms}$.

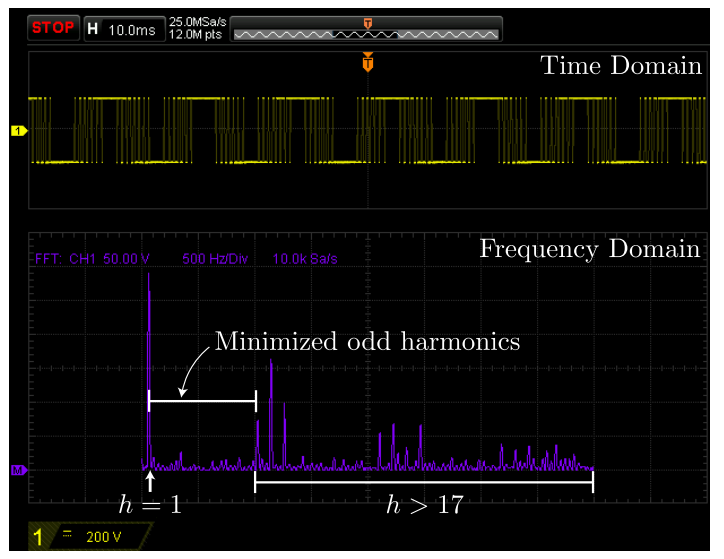

(b) The time and frequency-domain analysis of the two-level waveform confirms the minimization of the first seven odd harmonics.

Fig. 11: Experimental measurements of the computing circuit minimizing the first seven odd harmonics ( $h=3,5,7,9,11,13,15)$.

\section{2) Minimization of first seven odd nontriplen harmonics:}

In this verification test, the digital system is reprogrammed to minimize the first seven odd nontriplen harmonics ( $h=$ $5,7,11,13,17,19,23)$. Note that there are no modifications to the analog system of the computing circuit. A SPICE-based simulation of this scenario is shown in Fig. 12. The circuit converges to a KKT point in steady state (Fig. 12a), while the frequency domain analysis (Fig. 12c) again confirms that the specified harmonics are indeed minimized. The measurements from the hardware implementation of this scenario are shown in Fig. 13. In this case, we observe that the switching angle voltage variables converge to steady state in $3.7 \mathrm{~ms}$ (Fig. 13a). The time and frequency-domain analysis of the resulting inverter waveform in Fig. 13b confirms that the desired harmonics are minimized.

3) Minimization of first seven odd nontriplen harmonics with time varying modulation index command: In the third verification test, the digital system is programmed to minimize the first seven odd nontriplen harmonics while the commanded modulation index, $m$, is continually varied. Figure 14 illustrates the SPICE-based simulation for this scenario. As shown in Fig. 14a, $m$ is varied from 0 to 1 over $1 \mathrm{~s}$ from $t=0.2$ to $t=1.2$. Over this time period, the switching angle voltage variables (Fig. 14b) continuously evolve as the KKT 


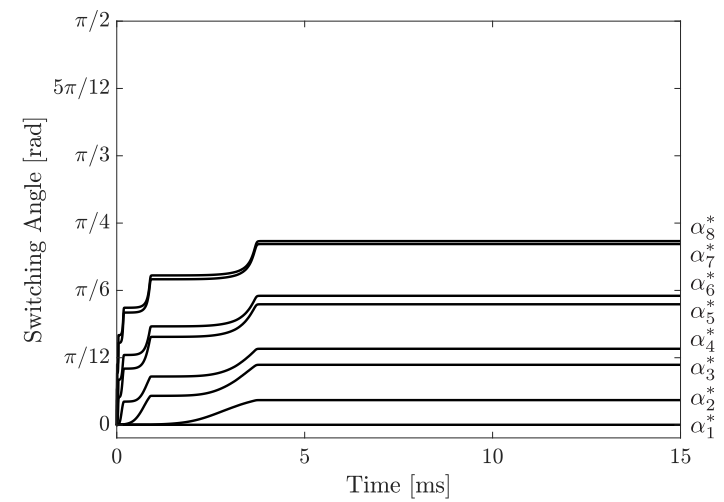

(a) The switching angle voltage variables $\alpha_{\ell}$ converge to the optimal value $\alpha_{\ell}^{\star}$ in approximately $3.8 \mathrm{~ms}$.

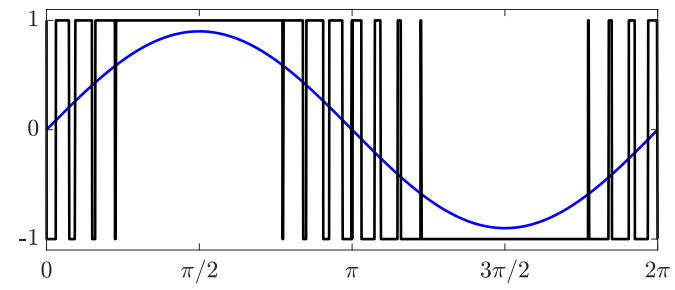

(b) The optimal quarter-wave symmetric two-level waveform obtained by using the computed switching angles $\alpha_{\ell}^{\star}$

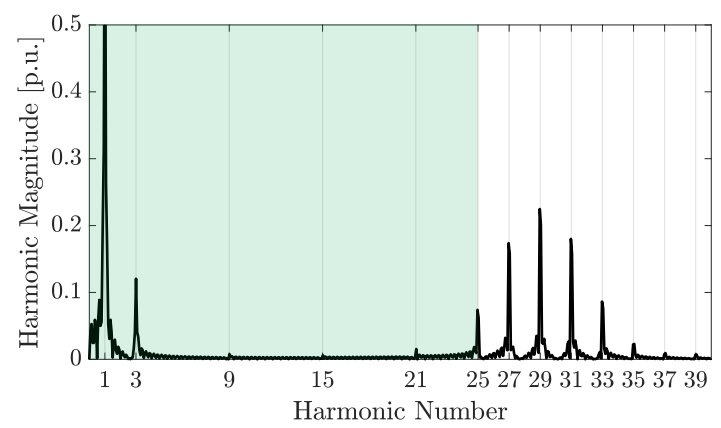

(c) A frequency-domain analysis of the two-level waveform confirms the minimization of the first seven odd nontriplen harmonics.

Fig. 12: Simulation of the computing circuit minimizing the first seven odd nontriplen harmonics ( $h=5,7,11,13,17,19,23)$.

points $\alpha_{\ell}^{\star}$ change as a function of $m$. Figure $14 \mathrm{c}$ shows a waterfall plot that analyzes the time-varying frequency content of the resulting two-level inverter waveform. As shown, the first seven odd nontriplen harmonics are indeed continually minimized over the entire simulation period, while the fundamental frequency $(h=1)$ increases in magnitude due to the control of the modulation index. This test validates the dynamic tracking capability of the proposed approach. As the commanded modulation index changes, the corresponding optimal switching angles will also change. Thus, conventional methods that rely on LUTs or ANNs are unable to address these scenarios unless the response is preprogrammed into the system. On the contrary, the proposed computing circuit accepts the commanded modulation index as an input, and the switching angle voltage variables automatically converge to the new optimal solution.

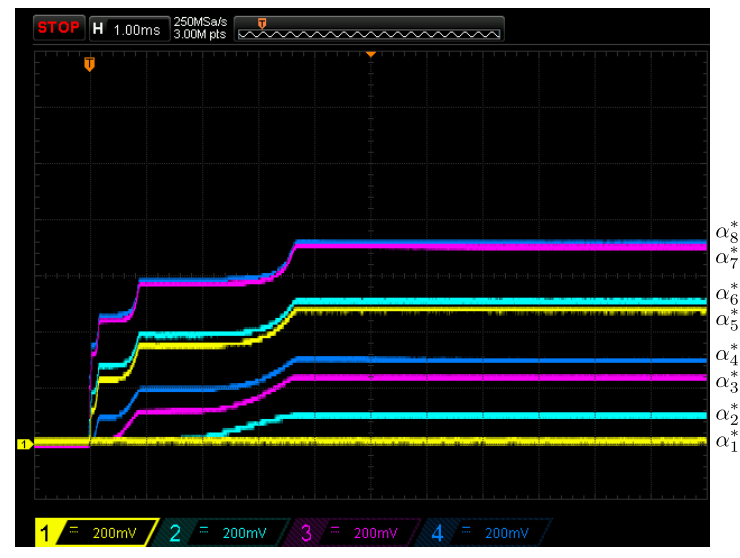

(a) Measurements of the switching angle voltage variables $\alpha_{\ell}$ converge to the optimal value $\alpha_{\ell}^{\star}$ in $3.7 \mathrm{~ms}$.

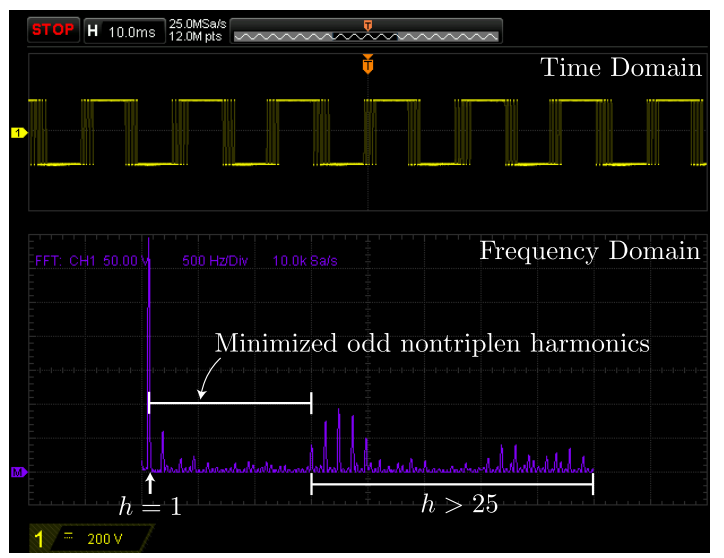

(b) The time and frequency-domain analysis of the two-level waveform confirms the minimization of the first seven odd nontriplen harmonics.

Fig. 13: Experimental measurements of the computing circuit minimizing the first seven odd nontriplen harmonics $(h=5,7,11,13,17,19,23)$.

\section{CONCluding Remarks AND Directions FOR FUTURE WORK}

This paper presented a hybrid analog/digital computing circuit capable of solving the selective harmonic minimization problem in a fast and power-efficient manner. The experimentally verified convergence speed $(<5.0 \mathrm{~ms})$ and power consumption $(750 \mathrm{~mW})$ of the computing circuit are substantially lower than previously published works where data is available. Moreover, the proposed circuit is capable of dynamically updating the optimization in real-time based on inputs and feedback-illustrated specifically in this paper by dynamic control of the modulation index-which, to the best of the authors' knowledge, is unique in the selective harmonic minimization literature.

There are a number of directions for future study. First, grid codes or uneven weighting function across the harmonics to be minimized could be incorporated by modifying the objective function. Second, while the experimental results shown here were targeted for a lower power single-phase inverter application, the proposed circuit could be generalized for other converter configurations that have been explored in the context of selective harmonic minimization techniques, such as three-phase, multi-level, or unbalanced converters. 


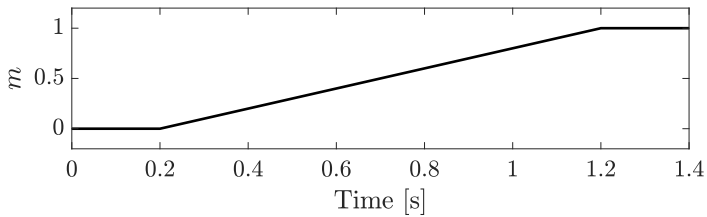

(a) The commanded modulation index, $m$, is varied from 0 to 1 from $0.2 \mathrm{~s}$ to $1.2 \mathrm{~s}$.

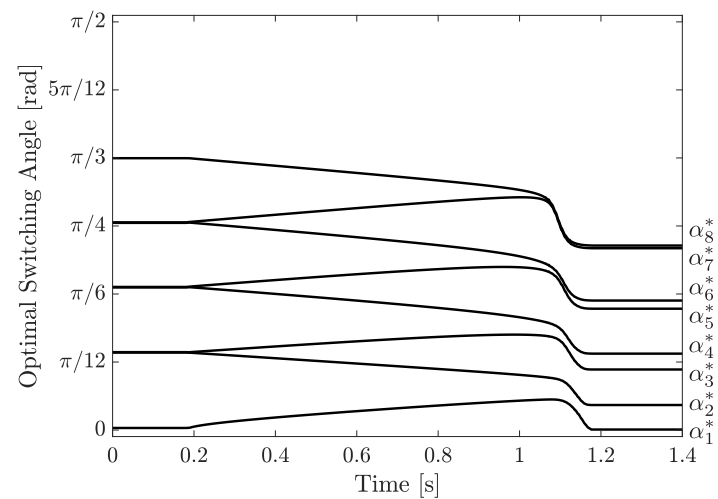

(b) The switching angle voltage variables $\alpha_{\ell}^{\star}$ continuously evolve in response to the changing modulation index, which changes the corresponding optimal solution.

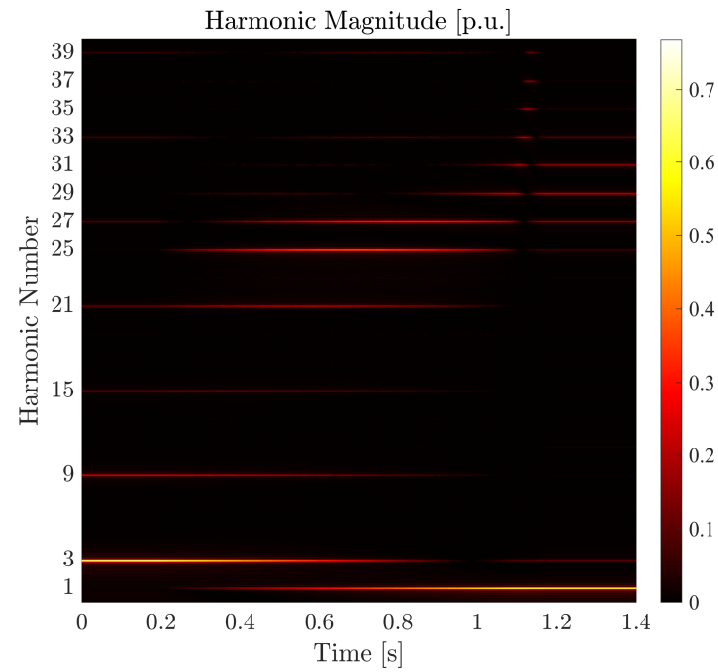

(c) A waterfall plot of the resulting two-level waveform shows that the first seven odd nontriplen harmonics are continually minimized, while the fundamental frequency ( $h=$ 1 ) increases in magnitude due to the control of $m$. The colors on the plot indicate the harmonic magnitude at the indicated harmonic number; the black color indicates that there is essentially no harmonic content at the particular frequency and time instance.

Fig. 14: Simulation of the computing circuit minimizing the first seven odd nontriplen harmonics ( $h=5,7,11,13,17,19,23)$ while dynamically controlling the modulation index, $m$.

Third, further study is required to precisely benchmark the performance of the proposed technique with existing methods for selective harmonic minimization, namely numericalbased optimization methods, with respect to speed and power consumption. This would potentially entail a register-transfer level (RTL) simulation of metaheuristic-based optimization techniques to provide a fair hardware comparison and accurate power estimate. Finally, alternative all-digital, all-analog, or other hybrid analog/digital configurations of the proposed computing circuit can be considered to determine the resulting trade-offs in speed, energy consumption, and cost for various applications.

\section{REFERENCES}

[1] J. Poon, M. Sinha, S. V. Dhople, and J. Rivas-Davila, "Real-time selective harmonic minimization using hybrid analog/digital computing," in 2021 IEEE Applied Power Electronics Conference and Exposition (APEC), 2021, pp. 1041-1046. DOI: 10 . 1109/ APEC42165 . 2021. 9487342.

[2] S. Vazquez, J. Rodriguez, M. Rivera, L. G. Franquelo, and M. Norambuena, "Model predictive control for power converters and drives: Advances and trends," IEEE Transactions on Industrial Electronics, vol. 64, no. 2, pp. 935-947, 2017. DOI: 10.1109/TIE.2016.2625238.

[3] S. Kouro, P. Cortes, R. Vargas, U. Ammann, and J. Rodriguez, "Model predictive control-A simple and powerful method to control power converters," IEEE Transactions on Industrial Electronics, vol. 56, no. 6 , pp. 1826-1838, 2009. DOI: 10.1109/TIE.2008.2008349.

[4] T. Geyer, G. Papafotiou, and M. Morari, "Model predictive direct torque control-part I: Concept, algorithm, and analysis," IEEE Transactions on Industrial Electronics, vol. 56, no. 6, pp. 1894-1905, 2009. DOI: 10.1109/TIE.2008.2007030.

[5] T. Faulwasser, A. Engelmann, T. Mühlpfordt, and V. Hagenmeyer, "Optimal power flow: An introduction to predictive, distributed and stochastic control challenges," at - Automatisierungstechnik, vol. 66, no. 7, pp. 573-589, Jul. 2018, ISSN: 0178-2312. DOI: $10.1515 /$ auto2018-0040. [Online]. Available: http://dx.doi.org/10.1515/auto-20180040.

[6] W. Huang, X. Pan, M. Chen, and S. H. Low, "DeepOPF-V: Solving AC-OPF problems efficiently," 2021. arXiv: 2103.11793 [eess.SY]

[7] L. Kamas and S. Sanders, "Parameter and state estimation in power electronic circuits," IEEE Transactions on Circuits and Systems I: Fundamental Theory and Applications, vol. 40, no. 12, pp. 920-928, 1993. DOI: $10.1109 / 81.269033$.

[8] N. Moehle and S. Boyd, "Optimal current waveforms for switchedreluctance motors," in 2016 IEEE Conference on Control Applications (CCA), 2016, pp. 1129-1136. DOI: 10.1109/CCA.2016.7587958.

[9] - "Maximum torque-per-current control of induction motors via semidefinite programming," in 2016 IEEE 55th Conference on Decision and Control (CDC), 2016, pp. 1920-1925. DOI: 10.1109/CDC.2016. 7798545.

[10] F. G. Turnbull, "Selected harmonic reduction in static dc-ac inverters," IEEE Transactions on Communication and Electronics, vol. 83, no. 73 pp. 374-378, 1964. DOI: 10.1109/TCOME.1964.6541241.

[11] H. S. Patel and R. G. Hoft, "Generalized techniques of harmonic elimination and voltage control in thyristor inverters: Part I-harmonic elimination," IEEE Transactions on Industry Applications, vol. IA-9, no. 3 , pp. 310-317, 1973. DOI: 10.1109/TIA.1973.349908.

[12] _ "Generalized techniques of harmonic elimination and voltage control in thyristor inverters: Part II-voltage control techniques," IEEE Transactions on Industry Applications, vol. IA-10, no. 5, pp. 666-673, 1974. DOI: 10.1109/TIA.1974.349239.

[13] I. J. Pitel, "Spectral errors in the application of pulsewidth modulated waveforms," IEEE Transactions on Industry Applications, vol. IA-17, no. 3, pp. 289-295, 1981. DOI: 10.1109/TIA.1981.4503943.

[14] J. R. Wells, B. M. Nee, P. L. Chapman, and P. T. Krein, "Selective harmonic control: A general problem formulation and selected solutions," IEEE Transactions on Power Electronics, vol. 20, no. 6, pp. 1337-1345, 2005.

[15] M. S. A. Dahidah, G. Konstantinou, and V. G. Agelidis, "A review of multilevel selective harmonic elimination PWM: Formulations, solving algorithms, implementation and applications," IEEE Transactions on Power Electronics, vol. 30, no. 8, pp. 4091-4106, 2015.

[16] J. Napoles, A. J. Watson, J. J. Padilla, J. I. Leon, L. G. Franquelo, P. W. Wheeler, and M. A. Aguirre, "Selective harmonic mitigation technique for cascaded H-bridge converters with nonequal dc link voltages," IEEE Transactions on Industrial Electronics, vol. 60, no. 5, pp. 1963-1971, 2013. DOI: 10.1109/TIE.2012.2192896.

[17] J. Chiasson, L. M. Tolbert, K. McKenzie, and Zhong Du, "A complete solution to the harmonic elimination problem," in Eighteenth Annual IEEE Applied Power Electronics Conference and Exposition, 2003. APEC '03., vol. 1, 2003, 596-602 vol.1. DOI: 10.1109/APEC. 2003. 1179273

[18] P. N. Enjeti, P. D. Ziogas, and J. F. Lindsay, "Programmed PWM techniques to eliminate harmonics: A critical evaluation," IEEE Transactions on Industry Applications, vol. 26, no. 2, pp. 302-316, 1990. DOI: $10.1109 / 28.54257$.

[19] J. R. Wells, B. M. Nee, P. L. Chapman, and P. T. Krein, "Optimal harmonic elimination control," in 2004 IEEE 35th Annual Power 
Electronics Specialists Conference (IEEE Cat. No.04CH37551), vol. 6, 2004, 4214-4219 Vol.6. DOI: 10.1109/PESC.2004.1354745.

[20] J. Pontt, J. Rodriguez, and R. Huerta, "Mitigation of noneliminated harmonics of SHEPWM three-level multipulse three-phase active front end converters with low switching frequency for meeting standard ieee-519-92," IEEE Transactions on Power Electronics, vol. 19, no. 6 , pp. 1594-1600, 2004. DOI: 10.1109/TPEL.2004.836616.

[21] J. Napoles, J. I. Leon, L. G. Franquelo, R. Portillo, and M. A. Aguirre, "Selective harmonic mitigation technique for multilevel cascaded $\mathrm{H}$ bridge converters," in 2009 35th Annual Conference of IEEE Industrial Electronics, 2009, pp. 806-811. DOI: 10.1109/IECON.2009.5415023.

[22] J. Sun, S. Beineke, and H. Grotstollen, "Optimal PWM based on realtime solution of harmonic elimination equations," IEEE Transactions on Power Electronics, vol. 11, no. 4, pp. 612-621, 1996.

[23] J. N. Chiasson, L. M. Tolbert, K. J. McKenzie, and Zhong Du, "A complete solution to the harmonic elimination problem," IEEE Transactions on Power Electronics, vol. 19, no. 2, pp. 491-499, 2004.

[24] C. Buccella, C. Cecati, M. G. Cimoroni, and K. Razi, "Analytical method for pattern generation in five-level cascaded H-bridge inverter using selective harmonic elimination," IEEE Transactions on Industrial Electronics, vol. 61, no. 11, pp. 5811-5819, 2014.

[25] K. Yang, Q. Zhang, J. Zhang, R. Yuan, Q. Guan, W. Yu, and J. Wang, "Unified selective harmonic elimination for multilevel converters," IEEE Transactions on Power Electronics, vol. 32, no. 2, pp. 1579-1590, 2017. DOI: 10.1109/TPEL.2016.2548080.

[26] A. Janabi, B. Wang, and D. Czarkowski, "Generalized Chudnovsky algorithm for real-time PWM selective harmonic elimination/modulation: Two-level VSI example," IEEE Transactions on Power Electronics, vol. 35, no. 5, pp. 5437-5446, 2020. DOI: 10 .1109/TPEL. 2019. 2945684.

[27] A. Kavousi, B. Vahidi, R. Salehi, M. K. Bakhshizadeh, N. Farokhnia, and S. H. Fathi, "Application of the bee algorithm for selective harmonic elimination strategy in multilevel inverters," IEEE Transactions on Power Electronics, vol. 27, no. 4, pp. 1689-1696, 2012.

[28] L. G. Franquelo, J. Napoles, R. C. P. Guisado, J. I. Leon, and M. A. Aguirre, "A flexible selective harmonic mitigation technique to meet grid codes in three-level PWM converters," IEEE Transactions on Industrial Electronics, vol. 54, no. 6, pp. 3022-3029, 2007.

[29] H. Taghizadeh and M. Tarafdar Hagh, "Harmonic elimination of cascade multilevel inverters with nonequal dc sources using particle swarm optimization," IEEE Transactions on Industrial Electronics, vol. 57, no. 11 , pp. $3678-3684,2010$.

[30] V. G. Agelidis, A. Balouktsis, and I. Balouktsis, "On applying a minimization technique to the harmonic elimination PWM control: The bipolar waveform," IEEE Power Electronics Letters, vol. 2, no. 2, pp. 41-44, 2004.

[31] M. S. A. Dahidah and V. G. Agelidis, "Selective harmonic elimination PWM control for cascaded multilevel voltage source converters: A generalized formula," IEEE Transactions on Power Electronics, vol. 23, no. 4, pp. 1620-1630, 2008.

[32] F. Filho, L. M. Tolbert, Y. Cao, and B. Ozpineci, "Real-time selective harmonic minimization for multilevel inverters connected to solar panels using artificial neural network angle generation," IEEE Transactions on Industry Applications, vol. 47, no. 5, pp. 2117-2124, 2011.

[33] F. Filho, H. Z. Maia, T. H. A. Mateus, B. Ozpineci, L. M. Tolbert, and J. O. P. Pinto, "Adaptive selective harmonic minimization based on ANNs for cascade multilevel inverters with varying dc sources," IEEE Transactions on Industrial Electronics, vol. 60, no. 5, pp. 1955-1962, 2013. DOI: 10.1109/TIE.2012.2224072.

[34] H. W. Kuhn and A. W. Tucker, "Nonlinear programming," in Proceedings of the Second Berkeley Symposium on Mathematical Statistics and Probability, Berkeley, Calif.: University of California Press, 1951, pp. 481-492.

[35] L. O. Chua and Gui-Nian Lin, "Nonlinear programming without computation," IEEE Transactions on Circuits and Systems, vol. 31, no. 2, pp. 182-188, 1984. DOI: 10.1109/TCS.1984.1085482.

[36] M. P. Kennedy and L. O. Chua, "Neural networks for nonlinear programming," IEEE Transactions on Circuits and Systems, vol. 35, no. 5, pp. 554-562, 1988. DOI: 10.1109/31.1783.

[37] G. Still, "Lectures on parametric optimization: An introduction," in Optimization Online, 2018.

[38] K. Yang, Q. Zhang, R. Yuan, W. Yu, J. Yuan, and J. Wang, "Selective harmonic elimination with groebner bases and symmetric polynomials," IEEE Transactions on Power Electronics, vol. 31, no. 4, pp. 2742-2752, 2016. DOI: 10.1109/TPEL.2015.2447555.

[39] K. Yang, Z. Yuan, R. Yuan, W. Yu, J. Yuan, and J. Wang, "A groebner bases theory-based method for selective harmonic elimination," IEEE
Transactions on Power Electronics, vol. 30, no. 12, pp. 6581-6592, 2015. DOI: 10.1109/TPEL.2014.2388077.

[40] V. G. Agelidis, A. I. Balouktsis, and M. S. A. Dahidah, "A five-level symmetrically defined selective harmonic elimination pwm strategy: Analysis and experimental validation," IEEE Transactions on Power Electronics, vol. 23, no. 1, pp. 19-26, 2008. DOI: 10.1109/TPEL.2007. 911770.

[41] F.-T. Lin, C.-Y. Kao, and C.-C. Hsu, "Applying the genetic approach to simulated annealing in solving some np-hard problems," IEEE Transactions on Systems, Man, and Cybernetics, vol. 23, no. 6, pp. 1752-1767, 1993. DOI: $10.1109 / 21.257766$.

[42] S. Tsutsui and A. Ghosh, "Genetic algorithms with a robust solution searching scheme," IEEE Transactions on Evolutionary Computation, vol. 1, no. 3, pp. 201-208, 1997. DOI: 10.1109/4235.661550.

[43] M. Tarafdar Hagh, H. Taghizadeh, and K. Razi, "Harmonic minimization in multilevel inverters using modified species-based particle swarm optimization," IEEE Transactions on Power Electronics, vol. 24, no. 10, pp. 2259-2267, 2009. DOI: 10.1109/TPEL.2009.2022166. 